\section{Another Approach to Proof: Arguments from Physics}

\author{
Gila Hanna, Toronto (Canada) \\ Hans Niels Jahnke, Essen (Germany)
}

\begin{abstract}
In the first part of the paper we will explore the use of arguments from physics in mathematical proof and give some reasons why this approach might be worthwhile. In the second part we will relate this idea to Freudenthal's concept of local organization. The third part of the paper will present the results of an empirical study conducted in Canada on the classroom use of arguments from physics in mathematical proof.
\end{abstract}

Kurzreferat: Im ersten Teil diskutieren wir den Gebrauch physikalischer Argumente in mathematischen Beweisen und begründen, warum dieser Zugang im Unterricht sinnvoll sein kann. Im zweiten Teil stellen wir eine Verbindung zu Freudenthals Begriff des lokalen Ordnens her. Der dritte Teil enthält Ergebnisse eines in Canada durchgeführten Unterrichtsversuchs zur Anwendung physikalischer Argumente bei mathematischen Beweisen.

ZDM-Classification: E50, G40, M10, M50

\section{An old and new approach to the teaching of proof}

Mathematicians often use arguments from physics in mathematical proofs. Some examples, such as the Dirichlet principle in the calculus of variations or Archimedes' use of the law of the lever for determining the volumes of solids, have become famous, and have in fact been regarded by the best mathematicians as elegant proofs, if not necessarily rigorous. It is only natural, then, that several authors, notably Polya and Winter (1978), have proposed that arguments from physics could and should be used in teaching school mathematics. Besides the publications of Polya (1981) and Winter (1978) there are a number of other papers and booklets with examples as Tokieda (1998) and Uspenskii (1961). Unfortunately, however, this approach to classroom teaching has not been sufficiently explored.

The application of physics under discussion here goes well beyond the simple physical representation of mathematical concepts, which of course has long proven its own usefulness in teaching. What is being explored here is the classroom use of proofs in which a principle of physics, such as the uniqueness of the centre of gravity, plays an integral role in a proof by being treated as if it were an axiom or a theorem of mathematics. This application of physics is also entirely distinct from experimental mathematics, which purports to employ empirical methods to draw valid general mathematical conclusions from the exploration of a large number of instances.

Let us look at a typical example. It is a well-known theorem of elementary geometry (the so-called Varignon theorem) that, given an arbitrary quadrangle $A B C D$, the midpoints of its sides $\mathrm{W}, \mathrm{X}, Y, Z$ form a parallelogram (see figure 3 below). A purely geometrical proof of this result would divide the quadrangle into two triangles and apply a similarity argument.
An argument from mechanics, on the other hand, would consider points $A, B, C, D$ as four weights, each of unit mass, connected by rigid but weightless rods. Of course such a system, with a total mass of 4 , has a centre of gravity, and it is this which we need to determine. The two sub-systems $A B$ and $C D$ each have weight 2 , and their respective centres of gravity are their midpoints $W$ and $Y$. From static considerations we may replace $A B$ and $C D$ by $W$ and $Y$, each having mass 2 . But $A B$ and $C D$ make up the whole system $A B C D$. Its centre of gravity is therefore the midpoint $M$ of $W Y$. In the same way we can consider $A B C D$ as made up of $B C$ and $D A$. Therefore the centre of gravity of $A B C D$ is must also be midpoint of $X Z$. Since the centre of gravity is unique, this midpoint must be $M$. This means that $M$ cuts both $W Y$ and $X Z$ into equal parts. Thus $W X Y Z$, whose diagonals are $W Y$ and $X Z$, is a parallelogram.

We can learn from this example that an argument from physics may add to our intuitive understanding of the mathematics involved. To an untutored mind it may seem rather surprising that every quadrangle, however irregular, has a property of such high regularity. When one considers the quadrangle as a four-point system of masses, however, it is immediately clear that its centre of gravity must also divide into equal parts the two levers that connect the midpoints of the opposite sides.

We have said quite deliberately that this proof adds something to our understanding of the theorem, not that it gives the "real" or the "best" explanation of it. Certainly the more common purely geometric proof also provides insight. We simply want to say that the argument from physics allows us to look at the theorem from a new and intuitively appealing point of view.

In many cases, arguments from physics are a way for the mathematician to produce a more elegant proof. This is as true today as it has been in history. Frequently such a proof may be illuminating in different ways. It may reveal the essential features of a complex mathematical structure, or point out more clearly the relevance of a theorem to other areas of mathematics or to other scientific disciplines. In some cases, too, using an argument from physics may also help create a "holistic" version of a proof, one that can be grasped in its entirety, as opposed to a necessarily elaborate and almost inscrutable mathematical argument.

Frequently, arguments from physics may help to generalize. Following the lines of our previous physical argument, for example, we can determine the centre of gravity not only for systems with four masses, but also for those with 3, 5, 6 and so forth. It is highly plausible that in these cases, too, one would be able to translate the respective statements about the centre of gravity into purely geometrical theorems. In the case of three masses, for example, such a theorem would say that in any triangle the three medians intersect in a single point and that this point of intersection divides each median in a ratio $2: 1$ (see below).

If we look at the educational context from a broader perspective, we can see several reasons why this approach to the teaching of proof should be further developed and tested. First of all, in most Western countries there is a trend away from using proof in the 
classroom. In our view, this trend threatens to undercut the educational value of mathematics teaching, since conveying to students the concept of mathematical proof is an important component of this value. One way to counter this unfortunate tendency is to introduce fresh and possibly more attractive approaches to the teaching of proof. Such new approaches, and especially the use of arguments from physics, might well motivate teachers to rethink their attitude to proof.

Another reason to pursue the use of arguments from physics is that present-day mathematical practice displays a significant emphasis on experimentation, and it is only right that this be reflected in the classroom by a similar emphasis on experimental mathematics. But it would be dangerous from an educational point of view if experimental mathematics were to be represented in the schools only by "mathematics with computers". Quite the contrary: under the heading of experimental mathematics, the curriculum should include a strong component devoted to the classical applications of mathematics to the physical world. In cultivating this type of mathematics, students and teachers should be guided by the question of how mathematics helps to explore and understand the world around us. With such an approach, the teaching of proof would be embedded in building models and in inventing arguments to answer the question "why". Both of these activities force one to think about the derivation of consequences from assumptions, or, in other words, about proof. Working in this way on the borderline between mathematics and physics, it would also become clear that we can not only apply mathematics to physics, but also very often use statements from physics for the derivation of mathematical theorems.

For many reasons physics, the discipline nearest to mathematics, has become less and less a required subject in our schools. To maintain meaningful and interdisciplinary mathematics teaching it may therefore become necessary to include some elementary physics in the mathematics curriculum. Of course this will have to be done carefully, bearing in mind the value of the manifold applications from the social sciences which have entered the curriculum in the last few decades. Nevertheless, we think that some adjustments to the curriculum will be necessary if we are to convey to students a more valid and balanced view of mathematics.

\section{The educational evolution of proof}

In our view, introducing concepts and arguments from physics into the teaching of geometry could significantly contribute to the development of students' understanding of proof. One of the most difficult problems faced by educators when they start doing proofs with their students is the systematic nature of Euclidean geometry. Today, of course, nobody would teach Euclidean geometry in an axiomatic way. Yet a closer analysis of geometry textbooks and the practice of teaching would show that Euclid's system is always present in the background. It determines to a large extent the sequence of theorems and the arguments students are allowed to use in a proof. Though they bring only a small part of it to the attention of their students, teachers nevertheless have a mental picture of a "grand theory". For the teacher, the main function of a proof is to incorporate a new theorem into this grand theory. The students know little or nothing of this grand theory, however, and so they must necessarily have a completely different image of the function of proof.

As a consequence of this deeply rooted problem, geometry is bound to appear arbitrary and dogmatic to many students. Why are they asked to prove the angle sum theorem, but are allowed to use facts about angles formed by parallel lines intersected by a third line, rather than vice versa? Most educators are aware of this fundamental difficulty, and it would seem to be one of the main reasons why in the last twenty years teachers have limited the role of proof in their teaching more and more. The justification for this reduced role offered by many educators is the mantra that the important thing is not to teach proof, but rather to develop a "culture of reasoning" in the classroom.

As is frequently the case with such "soft" slogans, this one is true and wrong at the same time. On the one hand it does take into account the undeniable fact that we cannot really teach systematic geometry in our schools. On the other hand, however, it ignores the reality that one cannot really discuss proof without discussing theories. The very notion of proof is tied to the notion of theory (and here we use "theory," of course, in the sense of "systematic structure"). Every proof is based upon an understanding, explicit or implicit, of what can be taken as given and what kinds of argument are acceptable, and these questions can be answered only in the context of a theory. Seen this way, it is clear that proof necessarily involves a formal element, and that providing a proof involves much more than setting out some everyday argument. Even more significant in the context of our discussion, perhaps, is that one cannot really understand the importance of proof or its educational value unless one understands that it is intimately tied to the idea of theory.

Most people would accept without elaboration the statement that we are having bad weather because of a drop in atmospheric pressure. This is a typical everyday explanation, and in everyday situations it might be sufficient. The difference between educated and uneducated people, however, is that the former, though they too would accept this explanation, are very conscious of the fact that it would need considerable elaboration, involving reference to a number of theories and laws, if we wanted to understand fully why it is raining today. What we are aiming for in the teaching of proof is precisely to develop this sense for the qualitative difference between scientific explanations and everyday arguments.

As we develop the notion of proof in the classroom, then, we must also develop the notion of a theory. In this connection we find it very useful to consider Freudenthal's concept of local organisation in geometry. He states that

“... in introductory geometry the student can be lead to learn to organize shapes and phenomena in space by means of geometrical concepts and their properties. At a higher level he should organize these concepts and their properties by means of logical relations. Above this level this relational system can become a subject of investigation" (Freudenthal 1973, 458). 
What Freudenthal had in mind is shown by his example of the theorem on the perpendicular bisectors of a triangle. It is not necessary, in his view, to give a complete proof that calls upon the entire (implicit) background of the Euclidean system, starting with the equidistance property of perpendicular bisectors and then progressing to the fact that they meet in one and the same point. Rather, one can concentrate on certain aspects which, for one reason or another, are of interest in the specific teaching situation, while taking other aspects for granted. Thus, local organization aims at the exploration of a certain configuration and not at establishing a deductive truth within a larger system. Of course, the things taken for granted in the "local organization" are consistent with the truths of the larger system and could be proved in it. But since the students do not have an idea of the larger system, we have to take this "local organization" itself as the theory with which we are dealing.

Thus, in line with Freudenthal's concept of "local organisation" we would propose a distinction between "large" and "small" theories. Instead of building a large theory (namely, Euclidean geometry) in the course of the curriculum, it seems to be more appropriate to work in several small theories around stimulating applications. The physical mechanisms described and analysed in elementary statics could provide fruitful examples of such small theories.

What does it mean to work in a "small theory"? We are well aware that students will always have a somewhat fragmented knowledge of geometry, because it has to be developed step by step and cannot be conveyed to them all at once by a simple verbal explanation. The idea of a "small theory" recognises that the same is true of their understanding of proof. As educators, we have to accept the fact that in the minds of the students there is no fixed (axiomatic) basis of argumentation, and that we cannot expect them to believe that there is a procedure called proof, superior to their intuition, which establishes the truth of a theorem.

But in that case what would a proof accomplish for the students? The answer is that a proof exhibits relations and dependencies between different facts or statements. We will explain this by a classic example. The angles in a circle theorem can be proved by recourse to properties of isosceles triangles. In the context of Euclidean geometry as a system, this means that we can add the angles in a circle theorem to the bag of true (proved) theorems, since we have already proved that the base angles in isosceles triangles are equal, and this, in turn, can be derived from another theorem, and so on.

In our teaching, for the reasons we have discussed, we cannot rely on such a long chain of deduction, and thus the proof must be made to mean something different to the students. They are working in the context of a small thematic unit on angles inscribed in circles, a context in which it is natural to consider that isosceles triangles can be inscribed in such circles. By a proof the students might understand establishing a logical connection between the invariance of the inscribed angles and the properties of isosceles triangles. These properties, then, "explain" the invariance of inscribed angles.

In regard to truth, of course, the proof wouldn't change the situation, since both facts, the invariance of the inscribed angles and the equality of the base angles in isosceles triangles, are equally plausible to the students. Nevertheless, the proof would enhance the certainty of the inscribed angle theorem in the sense that a counterexample to it would also be a counterexample to the equality of the base angles in isosceles triangles.

This also sheds some light on the relationship between proof and measurement (see Hanna \& Jahnke, 1996). Students are often required to measure the angles of triangles and work out their sum. After having found that they always get around $180^{\circ}$, they are told that this has to be proved mathematically in order to show that it is true "for all triangles". This pedagogically well-meant step is problematic, since it implies that we can arrive at a law valid for the empirical triangles on the paper in front of us by a purely deductive procedure, without any measurement. The fact is, however, that when we are dealing with empirical triangles we must insist that the angle sum property is true only because we have measured it, and not because we have proved it mathematically.

This having been said, it is also true that a mathematical proof of the angle sum theorem will enhance its empirical certainty, since it connects this theorem with some other statement (for example, a statement on angles at parallels cut by a straight line) which we can also establish by measurement. Taking this example, the statement on angles at parallel lines functions as an independent test of the angle sum theorem in triangles. If there is a counterexample to one of the theorems it will be relevant to both.

For the students, the epistemological situation is similar to that of a physicist. No physicist will believe in a statement simply because it has been proved mathematically. He will test it by measuring, of course. Mathematical proofs are essential in physics nevertheless, because they connect the empirical statements. A theory of physics is a network of measurements and laws connected by proof. The theory as a whole is tested by the system of all measurements taken as a whole, and therefore it has a status more certain than a single measurement could have, irrespective of how often it might have been performed. In educational terms, one could say that this method teaches geometry like a theory of physics.

What does the idea of local organization mean for the approach of using arguments from physics in mathematical proof? First of all, since students usually do not have the necessary background in physics, we have to start with building up such a background. This means, for example, that we introduce a unit on statics into geometry teaching. This would be a "small theory" of a mixed nature, since it comprise statements and principles from both geometry and physics. (In the $18^{\text {th }}$ century, in fact, people spoke of "mixed mathematics" in such cases.)

The facts of statics which would be taken for granted in this theory are empirical laws about the equilibrium of 1and 2-dimensional "bodies". As we have said, the empirical character of these laws does no harm to the idea of a mathematical proof, because it is the function of such a proof to establish theoretical connections among statements, whether the statements themselves are empirical or theoretical. 
In such a unit, geometrical facts would be derived from principles of statics. This is what we call "using arguments from physics within mathematical proof'. The students would learn that the physical idea of equilibrium has a purely geometrical aspect. Theorems which they had proved earlier within a geometrical setting would get a new meaning by being considered as facts within physics. In this way, by becoming conscious that geometrical theorems can be considered in different contexts and proved in different ways, students might come to reflect in a new and more adequate way on the notion of proof itself.

It can be hoped, too, that building up a small theory of mixed mathematics would have a useful side-effect. Students involved in such a process of theory building might more easily grasp the idea that theories are consciously constructed with definite aims in mind. In our case, of course, the aim is to foster an understanding of the equilibrium of physical bodies.

\section{The teaching unit}

The objective of the unit was to enable the students to use the lever principle and the concept of the centre of gravity to prove that the medians of a triangle are concurrent at a point of trisection and to prove the Varignon theorem. This unit, which consisted of two 75-minute classroom periods, was part of a larger section on deductive geometry. The concept of centre of gravity was introduced along with three postulates:

\section{Postulate 1: The lever principle}

Postulate 2: Any system of masses has only one centre of gravity

Postulate 3: In any system of masses, if any two individual masses are replaced by a single mass equal to the sum of the two masses positioned at the centre of gravity of the two masses, then the location of the centre of gravity of the total system of masses remains unchanged.

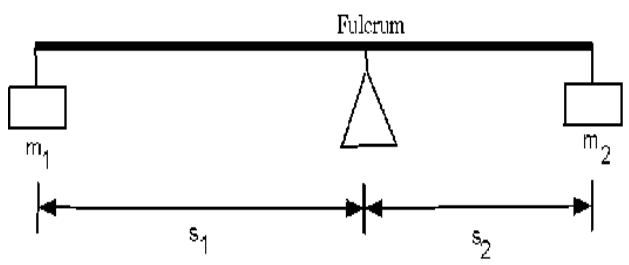

Figure 1: Balancing masses

The two 75 minute classroom periods introduced the three postulates mentioned above. At the commencement of the lesson, students were told briefly the purpose of the lesson, namely to learn some preliminary information on levers which they would need to know. As show in Figure 1 , the teacher demonstrated the balancing of various pairs of masses on a ruler supported at one point, and asked questions about the location or the weight of the masses.

The teacher also explained the relationship between the product of mass and distance from the fulcrum on each side of the fulcrum and indicated that this relationship is called the lever principle, and that the location of the balancing point or the fulcrum is also referred to as the centre of gravity. The students completed worksheets with diagrams of balanced masses, such as the one shown in Figure 1. The students also completed a table that showed that the ratio of masses was inversely proportional to the ratio of distances. In addition, they were asked questions such as the following:

In a system of a $200 \mathrm{~g}$ mass and a $300 \mathrm{~g}$ mass the $300 \mathrm{~g}$ mass is moved $4 \mathrm{~cm}$ closer to the centre of gravity, how must the $200 \mathrm{~g}$ mass be moved to maintain the same centre of gravity point? Explain using the lever principle.

\section{Medians of a triangle}

Part of the teaching unit was also devoted to the medians of a triangle. The objectives were 1) to have the students discover that the medians of a triangle intersect at a single point (the centre of gravity of the triangle when three equal masses are placed at its vertices) and that this point is located on a median, two-thirds of the way from each vertex, and 2) to have the students provide a proof or explanation why this must be so, using the three postulates related to the centre of gravity of a system.

The students worked in independent groups of three with a minimum of guidance from the teacher. Each group was provided with a clear acrylic triangle, a washable marker, a ruler and a number of $200 \mathrm{~g}$ masses (see Figure 2). The triangles were of varying shapes and sizes, including acute, obtuse and right triangles. Each triangle had six string loops taped to the corners of the triangles and at the midpoints of the sides. The students were asked to find the balancing point with the masses suspended from the vertices of the triangle, and then to verify that this point remained unchanged when two masses were relocated from their respective vertices to the midpoint of the side joining the vertices where the masses came from.

The teacher gave some suggestions about balancing the triangles on the retort rod (Figure 2) and answered some of the questions that students asked as their work progressed. In the course of doing the experiment and answering the related questions in the worksheets, the students would have observed and been made aware of postulates 2 and 3 above. Included in the worksheets were questions requiring the students to explain or prove the properties of the medians of a triangle using these postulates.

At the end of the period, the students were asked to complete the worksheets and hand them in the following day. They were asked to prove that the three medians of a triangle are concurrent (intersect in a single point) and that the point of intersection divides each median in a ratio $2: 1$, once 
using traditional geometrical arguments and then using arguments from physics.

For the proof using arguments from physics they were told that "given that $\mathrm{D}, \mathrm{E}$ and $\mathrm{F}$ are the midpoints of their respective sides of triangle $\mathrm{ABC}$, prove the triangle medians theorem using physical reasoning by applying the lever principle (LP), Substitution Principle (SP) and existence and uniqueness of the centre of gravity (UCG). Hint: locate the centre of mass on each of the three medians."

\section{The Varignon Theorem}

The worksheet restated that two systems of masses are considered equivalent if both systems have the same centre of gravity and one system can be obtained from the other by relocating the individual masses while maintaining the centre of gravity. Further it reminded the students that Postulate 3 describes how one system of masses can be changed to another equivalent system. An example of two equivalent systems is a triangle with equal masses located at its vertices and the same triangle with one mass at a vertex and the other two at the midpoint of the opposite side of this vertex.

The students were given the following: Let $\mathrm{W}, \mathrm{X}, \mathrm{Y}$ and $\mathrm{Z}$ be the midpoints of the sides of any quadrilateral

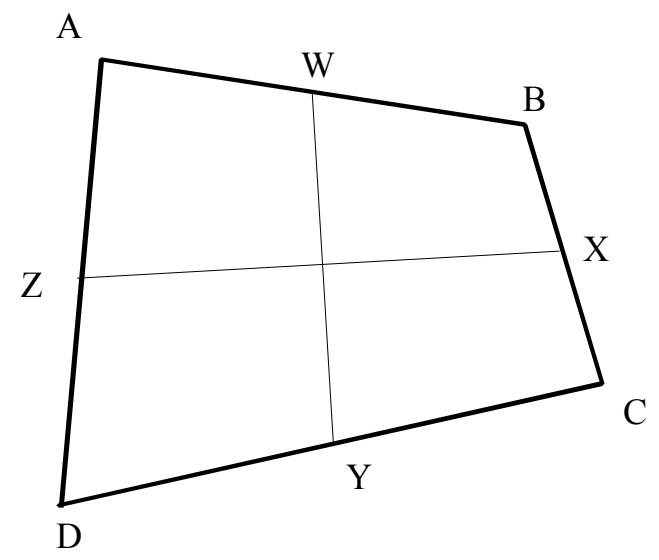

Figure 3: Quadrilateral ABCD with midpoints W, X, Y,

$\mathbf{Z}$

ABCD (Figure 3.), prove that the WXYZ is a parallelogram. They were also told to assume that equal masses are placed at the corners of the quadrilateral and use an argument from physics to prove (explain) by relocating the masses to make equivalent systems that the centre of mass of the quadrilateral is at the midpoint of $\mathrm{XZ}$.

\section{Student performance}

\section{The medians of a triangle}

As mentioned above, the students were asked to complete two proofs of the theorem that the medians of a triangle are concurrent at a point of trisection. One proof was to be based upon arguments from physics, while the other was to be a traditional geometric proof. These proofs were assigned as homework, and were handed in with the other worksheets before the questionnaire was administered and the interviews carried out. The assigned proofs were phrased as follows:

Geometry 1: Using geometrical arguments, given $\triangle \mathrm{ABC}$ with medians $\mathrm{BE}$ and $\mathrm{CF}$ intersecting at $\mathrm{G}$.

a) Prove $\mathrm{EF}: \mathrm{CB}=1: 2$

b) Prove EF $\| \mathrm{CB}$

c) Prove $\triangle \mathrm{EFG} \sim \triangle \mathrm{BCG}$

d) Prove EG:GB = FG:GC = 1:2

The worksheet indicated that for the geometric proof the students were to "use the theorems developed in class (chapter 5 of your textbook) to prove the medians of triangles theorem.

Physics 2a: Using arguments from physics write out a complete proof, applying the above postulates, explaining how experiment II proves the medians of a triangle all intersect at one point.

Physics 2b: What additional property does experiment II prove concerning the intersection of the medians of a triangle? Give reasons for your answer

The students' performance on these questions was rated by the classroom teacher in three categories: Correct response, partially correct response (including incomplete responses with correct start or correct use of arguments but still unfinished responses) and incorrect response (including responses with minor correct parts but mostly wrong).

Table 1. Assessment of students' proofs that the medians of a triangle are concurrent using physical or geometrical arguments $(N=25)$

\begin{tabular}{|c|c|c|c|}
\hline Question & Correct & Partial & Incorrect \\
\hline Physics 2a & 12 & 9 & 4 \\
\hline Physics 2b & 2 & 8 & 15 \\
\hline Geometry 1 & 23 & 1 & 1 \\
\hline
\end{tabular}

As shown in Table 1, the proof using arguments from physics that the medians of a triangle are concurrent (Physics 2a) was done correctly by 12 students and partially correctly by nine; four students produced proofs judged to be incorrect. Only two students provided correct answers to Physics $2 b$, in which they were to identify that the medians meet at the point of their trisection, with only eight providing partially correct ones.

This result stands in contrast to a much higher rate of success on the geometric proof (question Geometry 1), where all but two students produced a correct proof. One should note, however, that this question was accompanied by significant hints to the students on how to proceed, in the form of two diagrams and an explicit presentation of 
sub-steps (a) to (d), as shown above. No comparable guidance was provided to the students as to how to proceed with their first assignment, the proof using principles from physics. It also seems fair to assume that these results reflected previous classroom work, in which the students had become quite accustomed to the geometric approach. Indeed, the participating students had already had fourteen 75-minute assignment, the proof using principles from physics. It also seems fair to assume that these results reflected previous classroom work, in which the students had become quite accustomed to the geometric approach. Indeed, the participating students had already had fourteen 75-minute periods of traditional geometry in their Grade 12 class before the physics unit was introduced, and had also had some traditional geometry (angle theorems and congruence postulates) in Grade 10.

The expectation in question Physics $2 b$ was that the students would realise that the additional property sought is that the medians intersect in a ration of $1: 2$, a property that had already been covered in the classroom. It was also hoped that they would use the postulates to justify this property, rather than simply basing it upon their experimental observations. As a matter of fact, however, $60 \%$ of the students ( 15 out of 25 ) were unable to answer this question correctly. Eight students were on the right track but did not complete their proof, and only two students successfully answered this question (see appendix for an example of a correct response).

This disappointing outcome might well be a sign that the students did not completely understand the difference between (1) a proof that uses arguments from physics and (2) an informal justification based upon the results of an experiment. Indeed, the responses to the questionnaire revealed nothing to show that any of the students realised that the experiments they performed were not an integral part of the proof from physics, while there were several comments indicating that students did think they were. Among them were comments such as "The argument is convincing because you can visually see the answer;" "Because we did it ourselves;" "The physics proof assisted in understanding the geometric proof because when you see something you tend more to believe it;" and "Physics is more hands on where mathematics you have to visualize an physics is in front of you."

\section{The Varignon Theorem}

The students were asked to complete two proofs of the Varignon theorem. One proof was to be based upon arguments from physics, while the other was to be a traditional geometric proof. Both proofs were assigned as homework and were phrased as follows:

Physics 4a) Prove (explain) by relocating the masses to make equivalent systems that the centre of mass of the quadrilateral is at the midpoint of $\mathrm{XZ}$

Physics 4b) Prove that the centre of gravity is at the midpoint of WY.

Physics 4c) What do a) and b) say about the intersection of XZ and WY?

Physics 4d) Explain why WXYZ is a parallelogram.

Geometry 5) Without referring to concepts of physics, prove that $\mathrm{WXYZ}$ is a parallelogram by proving its sides are parallel to the diagonals of quadrilateral ABCD

6. Discuss which proof you find easier to do or understand, the one in question 4 (physics) or the one in question 5 (geometry).

Table 2. Assessment of students' proofs of the Varignon theorem using physical or geometrical arguments $(N=25)$

\begin{tabular}{|l|c|c|c|}
\hline \multicolumn{1}{|c|}{ Question } & Correct & Partial & Incorrect \\
\hline Physics 4a & 23 & 2 & -- \\
\hline Physics 4b & 23 & 2 & -- \\
\hline Physics 4c & 22 & -- & 3 \\
\hline Physics 4d & 21 & -- & 4 \\
\hline Geometry 5 & 16 & 5 & 4 \\
\hline
\end{tabular}

Almost all the students were able to give correct answers to the first four questions. Of the 22 students who answered $4 \mathrm{c}$ correctly, 10 made the observation that $\mathrm{XZ}$ and WY bisect each other, whereas the other 12 said that $\mathrm{XZ}$ and WY intersected at the centre of gravity. Though question $4 \mathrm{~d}$ was answered correctly by as many as 21 students, only three students were able to base their correct answer on the observation that the diagonals of WXYZ, XZ and WY, bisected each other; the remaining 18 students went on to give a full geometric proof.

The following are examples of correct answers:

Physics 4a. "According to postulate 3, if the masses at $A$ and $D$ are moved to $Z$, and the masses at $B$ and $C$ are moved to $X$, then the system will balance. The centre of gravity, therefore, will be the midpoint of $X Z$, as that is the point where the mass $x$ distance product will be equal for both sides."

Physics 4b. "Similar to part a), if the masses from $A$ and $B$ are moved to $W$, and the masses from $C$ and $D$ are moved to $Y$, the system will balance, meaning the centre of gravity must be equidistant from each pair of masses, which would be the midpoint of WY."

In the case of question Physics 4c, where students were asked "What do a) and b) say about the intersection of $\mathrm{XZ}$ and WY?", only 10 students stated correctly that "XZ and WY bisect each other", whereas 12 students gave a partially correct answer saying that " $X Z$ and $W Y$ intersect at the centre of gravity" without mentioning the crucial fact of bisection.

Of the 21 students who answered Physics 4d correctly, only three were able to state that it is because "WY and $Z X$, the diagonal of $W X Y Z$ bisect each other" and thus base their answer on the argument from physics. As many as 18 students saw it necessary to give a full geometric proof, showing that since the opposite sides are equal, $\mathrm{ZW}=\mathrm{YX}$ and $\mathrm{ZY}=\mathrm{WX}$, WXYZ must be a parallelogram.

\section{Students' responses to questions about the two methods} of proof

In the questionnaire mentioned earlier, the participants were asked to respond to and comment on a range of questions including the following:

1. Is the argument from physics convincing? 
2. Which proof, the geometric or the one based on arguments from physics, was clearer?

3. Which proof was easier to remember?

4. Does everyday experience with balancing things make the argument from physics clearer?

The first four questions were related to proofs that the medians of a triangle are concurrent whereas the last question was related to the Varignon theorem. The responses and comments indicated that the idea of using arguments from physics has a great deal of appeal for students in the case of the medians of a triangle theorem. An important specific finding of this study is that all but two of the participants found the proof using the argument from physics convincing, as shown in Table 2.

\begin{tabular}{|l|l|l|l|}
\hline 1. Is the argument from physics convincing? & Yes (23) & No (1) & $?(1)$ \\
\hline 2. Which proof is clearer? & Physics (15) & Geom. (9) & $?(1)$ \\
\hline 3. Which proof is easier to remember? & Physics (15) & Geom. (5) & $?(5)$ \\
\hline 4. Does everyday experience with balancing help? & Yes (17) & No (2) & $?(6)$ \\
\hline 5. In the case of Varignon, which proof is easier? & Physics (4) & Geom. (14) & $?(7)$ \\
\hline
\end{tabular}

Table 3. Students' responses to four questionnaire questions $(N=25)$

Note: "?" means "no response" or "ambiguous response"

As mentioned, the students were also given space in the questionnaire to add a few sentences of their own in response to each question, and were also interviewed. Their free-format responses give further insight into the issue of "convincing". One student remarked that "the argument from physics actually made sense," and another responded that in using physics "there is a hands-on, 3-D, example of why the argument is true." Yet another student remarked that "it is difficult to see how the physics argument is related to the issue at hand earlier on, but the connection eventually becomes more evident and concrete than a geometric proof could ever be."

As responses to questions 2 and 3 in Table 3 also show, $60 \%$ of the participating students judged the proof using concepts and principles from physics to be clearer and easier to remember than the corresponding geometric proof. Nine of the 25 participants ranked the geometrical proof higher in terms of clarity, and five of them thought the geometric proof was easier to remember.

It should be pointed out that the 15 students who chose the proof using physics as being clearer were not all the same as the 15 who thought it easier to remember. In other words, judging a proof to be clearer did not mean judging it to be easier to remember. In fact, three of the nine students who thought the geometric proof was clearer found the physics proof to be easier to remember, while one student who thought the physics proof was clearer nevertheless thought the geometric proof was easier to remember.

For some students, hands-on experience seemed to have been the key factor in ease of retention. As one student put it in the course of an interview, the proof with physics was easier to remember "because you are actually doing it." Another student gave a similar explanation for the same conclusion: "You actually did it yourself. In math the teacher is just explaining it to you. Hands on sticks with you more."

Responses to the case of the Varignon theorem, question 5 in Table 3, indicated that as many as 14 students thought that the geometric proof was the easier one, while 4 students found the physics proof easier and 7 students did not respond. This is somewhat surprising in view of the students' better performance on the proof based on arguments from physics (see Table 2). Of the 14 students who found the geometric proof easier to do or understand, 8 offered additional comments. Three said that the geometrical proof was easier because of previous experience with geometry: "it is easier because I have more experience with it." " ... because it adheres more closely with a straightforward definition of the parallelogram." "I like it better because there was less to do."

Five students said that their lack of familiarity with physics concepts was an impediment to proving using arguments from physics. Typical comments were: "I find the geometrical proof easier. It is provable geometrically, whereas the other is too conceptual." or "I find it easier to understand without referring to concepts of physics. I need to have a clear understanding of the postulates before I can do the question. It is more difficult to visualize the concepts."

Two of these students even found the ideas from physics somewhat cumbersome. One said: "The geometric proof is easier to understand since you don't have to think about centre of gravity and making sure that it is in the right place where it balances out." and the other: "I like the geometric one better because it is easier for me to visualise. I can test my answer by manipulating it on paper. But in the physics proof, I would have to assemble an apparatus etc. which I would not be able to get a hold of most of the time. I also find that the geometric proof is easier to explain."

Of the four students who found the proof using arguments from physics easier to do or understand, three gave the following reasons: 
"I find the proof in question 4 [using arguments from physics] easier to understand because it is more visual than the one in question 5[the geometric one]."

"Both of these proofs were easy to understand. However, I found the one in question 4 [using arguments from physics]much easier to see, to quickly identify. The proof in number 5 took a bit for me to see."

"I find the physics proof easier to understand because we've seen a physical proof of the reasoning. However, I prefer to work with the geometric one."

\section{Conclusions}

From the students' comments and the assessment of questions Physics $2 \mathrm{a}$ and $2 \mathrm{~b}$, then, it seems quite clear that many students felt that the experiments were part of the proof. These rudimentary experiments had been introduced into the teaching unit to establish in the students' minds the empirical plausibility of the physics principles used in the proof as well as of the conclusion of that proof. In this the experiments would seem to have been successful. They also appear to have been successful in conveying to the students the more general idea that concepts and principles from physics can be used in proving mathematical theorems.

Nevertheless, it is clear from the students' responses that most of them found the proof from physics to be convincing, as well as clearer and more readily remembered than the geometric proof. It is also clear that students saw these proofs as another way of looking at geometrical theorems. The results of this experimental teaching unit do support the tentative conclusion that teaching mathematical proofs using concepts and principles of physics is a promising pedagogical approach worthy of further exploration.

\section{Acknowledgements}

Preparation of this paper was supported in part by the Social Sciences and Humanities Research Council of Canada. We wish to acknowledge the cooperation of the students and the teacher who participated in this study.

\section{References}

Freudenthal, H. (1973). Mathematics as an Educational Task. Dordrecht: Reidel

Hanna, G. \& Jahnke, H. N. (1996). Proof and proving. In: Bishop, A.; Clements, K.; Keitel, C.; Kilpatrick, J.; Laborde, C.: International handbook of mathematics education, Dordrecht: Kluwer 1996, 877 - 908

Hanna, G. \& Jahnke, H. N. (1999). Using arguments from physics to promote understanding of mathematical proofs. In O. Zaslavsky (Ed.), Proceedings of the twenty-third conference of the international group for the psychology of mathematics education, Vol. 3, 73-80. Haifa: Israel.

Polya, G. (1981). Mathematical discovery: On understanding, learning and teaching problem solving ( 2 vols.; combined ed.). New York: John Wiley \& Sons.

Tokieda, T. F. (1998). Mechanical Ideas in Geometry. American Mathematical Monthly 105, $697-703$.

Uspenskii. V. A. (1961). Some Applications of Mechanics to Mathematics. New York: Balisdell Publishing Company.

Winter, H. (1978). Geometrie vom Hebelgesetz aus -- ein Beitrag zur Integration von Physik- und Mathematikunterricht der Sekundarstufe I. Der Mathematikunterricht 24, 5, 88-125.

\author{
Authors: \\ Gila Hanna, Ontario Institute for Studies in Education of \\ the University of Toronto, 252 Bloor Street West, \\ Toronto, Ontario, M5S 1V6. (Canada) \\ E-mail: mailto:ghanna@,oise.utoronto.ca \\ Hans Niels Jahnke, FB6: Mathematik und Informatik, \\ Universität Essen, D-45117 Essen (Germany) \\ E-mail: mailto:njahnke@,uni-essen.de
}

